Pacific Journal of Mathematics

COUNTABLE BOOLEAN ALGEBRAS AS SUBALGEBRAS AND
HOMOMORPHS 


\title{
COUNTABLE BOOLEAN ALGEBRAS AS SUBALGEBRAS AND HOMOMORPHS
}

\author{
T. E. Cramer
}

The problem of classifying all countable Boolean algebras appears to impossible to solve. This paper considers the following problem. Given a class $\mathscr{C}$ of countable Boolean algebras, which is closed under isomorphisms, characterize the classes of

(i) all Boolean algebras which have subalgebras in $\mathscr{C}$;

(ii) all subalgebras af members of $\mathscr{C}$;

(iii) all homomorphs of merhers of $\mathscr{C}$;

(iv) all Boolean algebras which have homomorphs in $\mathscr{C}$.

Definitive characterizations are obtained for the first three classes (Theorems 7,8 , and 9 ), and a representation of the last class is obtained when $\mathscr{C}$ is the class of all countable Boolean algebras (Theorem II).

Given a Boolean algebra $A$, let $X$ be the corresponding Boolean space. Define inductively: $D_{0}(X)$ as $X ; D_{1}(X)$ as the complement of the isolated points in $X$; for any ordinal $\alpha, D_{\alpha+1}(X)$ as $D_{1}\left(D_{\alpha}(X)\right)$; and for a limit ordinal $\alpha, D_{\alpha}(X)$ as $\cap\left\{D_{\beta}(X): \beta<\alpha\right\}$. Then $D_{\alpha}(X)$ is a closed subspace of $X$ for each ordinal $\alpha$. The Boolean algebra $A$ is said to be siperatomic if $D_{\alpha}(X)=\varnothing$, for some ordinal $\alpha$. If $\gamma$ is the last ordinal for which $D_{r}(X)=\varnothing$, the cardinal sequence $\Gamma(A)$ of the superatomic Boolean algebra $A$ is defined in [1] as the sequence of order type $\gamma$ whose $\alpha$-term is the cardinality of the set of isolated points of $D_{\alpha}(X), \alpha<\gamma$. Note that each term of $\Gamma(A)$ is infinite except for the $\gamma-1$ term, which must be finite.

If $\mathscr{S}$ is the class of all superatomic Boolean algebras, the class $\{\Gamma(A): A \in \mathscr{S}\}$ may be partially ordered by setting $\Gamma(A) \leqq \Gamma(B)$ when the order type of $\Gamma(A)$ is not larger than that of $\Gamma(B)$, and when the $\alpha$-term of $\Gamma(A)$ is not larger than the $\alpha$-term of $\Gamma(B)$. It is shown in [4] that every countable superatomic Boolean algebra has as its Boolean space the ordinal $\omega^{\alpha} n+1$ endowed with the order topology, for some countable ordinal $\alpha$ and natural numbers $n$. Thus the relation "@" well orders the class of cardinal sequences of countable superatomic Boolean algebras.

Lemma 1. If $X$ and $Y$ are Boolean spaces, and $\theta$ is a continuous function from $X$ onto $Y$, then $\theta\left[D_{\alpha}(X)\right]$ contains $D_{\alpha}(Y)$, for each ordinal $\alpha$.

Proof. Assume that $\alpha=1$, and that $y$ is an element of $Y-$ 
$\theta\left[D_{1}(X)\right]$. Then $X-\theta^{-1}(y)$ is closed because $\theta^{-1}(y)$ is the union of isolated points. Therefore, $\theta\left[X-\theta^{-1}(y)\right]=Y-\{y\}$ must be closed, so $y$ is isolated and does not belong to $D_{1}(Y)$.

Now assume that $\theta\left[D_{\alpha}(X)\right]$ contains $D_{\alpha}(Y)$, for some ordinal $\alpha$. Then $\theta\left[D_{\alpha+1}(X)\right]=\theta\left[D_{1}\left(D_{\alpha}(X)\right)\right] \supseteqq D_{1}\left(D_{\alpha}(Y)\right)=D_{\alpha+1}(Y)$.

Finally, assume $\theta\left[D_{\alpha}(X)\right] \supseteqq D_{\alpha}(Y)$ for each $\alpha<\beta, \beta$ some limit ordinal. Let $y$ be a element of $D_{\beta}(Y)$, and define $K_{\alpha}=D_{\alpha}(X) \cap\left[\theta^{-1}(y)\right]$. By hypothesis, $K_{\alpha}$ is closed and nonempty for each $\alpha<\beta$. Since $X$ is compact, $\cap\left\{K_{\alpha}: \alpha<\beta\right\} \neq \varnothing$, so let $x$ be a point in this intersection. Then $x$ is in $\cap\left\{D_{\alpha}(X): \alpha<\beta\right\}$ and is in $\theta^{-1}(y)$, so $\theta\left[D_{\beta}(X)\right] \supseteqq$ $D_{\beta}(Y)$.

Lemma 2. If $X$ is a Boolean space and $Y$ is a clopen subspace of $Y$, then $D_{\alpha}(X) \cap Y=D_{\alpha}(Y)$ for every ordinal $\alpha$.

Proof. By induction.

Theorem 3. If $A$ and $B$ are superatomic Boolean algebras, $A$ is countable, and $\Gamma(A) \leqq \Gamma(B)$, then there is a monomorphism from $A$ to $B$.

Proof. Since $A$ is a countable superatomic Boolean algebra, its Boolean space can be represented as $\omega^{\alpha} n+1$ with the order topology, for some countable ordinal $\alpha$, and some natural number $n$. Let $Y$ be the Boolean space of $B$. The dual statement to the theorem will be proved by constructing a continuous mapping of $Y$ onto $\omega^{\alpha} n+1$. Two topological observations will be needed.

(1) Suppose that $\left\{\left(Y_{\beta}, X_{\beta}\right): \beta<\alpha\right\}$ is a set of pairs of topological spaces. If there are continuous functions from $Y_{\beta}$ to $X_{\beta}$, for all $\beta<\alpha$, then there is a continuous function from the disjoint union $\cup\left\{Y_{\beta}: \beta<\alpha\right\}$ to the disjoint union $\cup\left\{X_{\beta}: \beta<\alpha\right\}$.

(2) Suppose that $Y$ is a topological space with an open subspace $U$. If $f$ is a continuous function from $U$ to a topological space $X$, and $f^{-1}(V)$ is closed in $Y$ whenever $V$ is closed and compact in $X$, then there is a continuous extension $g$ of $f$ from $Y$ to the one-point compactification of $X$. Define $g(y)=f(y)$ when $y \in U$, and $g(y)=x_{0}$ when $y \in Y-U$, where $x_{0}$ is the compactification point.

The proof proceeds by inducting first on $\alpha$ and then on $n$. If $a=0$ then the Boolean space of $A$ is finite and has the discrete topology. Since $\Gamma(A) \leqq \Gamma(B)$, the cardinality of $Y$ is at least as large as that of the Boolean space of $A$, so an application of (1) produces the desired function between these spaces.

If $\alpha=\beta+1$ for some ordinal $\beta$, and $n=1$, then $D_{\beta}(Y)$ is in- 
finite. Pick a sequence $\left\{y_{n}: n<\omega\right\}$ of distinct points in $D_{\beta}(Y)$, and choose disjoint clopen sets $\left\{Y_{n}: n<\omega\right\}$ in $Y$ such that $y_{n} \in Y_{n}$, for each $n<\omega$. Lemma 2 implies that $D_{\beta}\left(Y_{n}\right) \neq \varnothing$ for each $n<\omega$, when $Y_{n}$ is considered as a Boolean space. Write $\omega^{\beta+1}+1$ as

$$
\left[0, \omega^{\beta}\right] \cup\left[\omega^{\beta}+1, \omega^{\beta} 2\right] \cup \cdots \cup\left\{\omega^{\beta+1}\right\} .
$$

Each subset $\left[\omega^{\beta} n+1, \omega^{\beta}(n+1)\right]$ is homeomorphic to $\omega^{\beta}+1$, so the induction hypothesis insures a continuous mapping of $Y_{n}$ onto

$$
\left[\omega^{\beta} n+1, \omega^{\beta}(n+1)\right], n<\omega .
$$

Apply (1) and then (2).

Assume now that $\alpha$ is a limit ordinal and $n=1$. Choose a strictly increasing sequence $\alpha(0), \alpha(1), \alpha(2), \ldots$ converging to $\alpha$. Pick points $y_{n}$ in $D_{\alpha(n)}(Y)-D_{\alpha(n+1)}(Y), n<\omega$, and let $\left\{Y_{n}: n<\omega\right\}$ be a set of disjoint clopen sets in $Y$ such that $y_{n} \in Y_{n}, n<\omega$. Represent $\omega^{\alpha}+1$ as $\left[0, \omega^{\alpha(0)}\right] \cup\left[\omega^{\alpha(0)}+1, \omega^{\alpha(1)}\right] \cup \cdots \cup\left\{\omega^{\alpha}\right\}$. As before, there must be continuous functions from $Y_{n}$ onto $\left[\omega^{\alpha(n)}+1, \omega^{\alpha(n+1}\right]$, and a continuous extension from $Y$ onto $\omega^{\alpha}+1$.

Finally, if $n>1, D_{\alpha}(Y)$ contains at least $n$ points. Pick a clopen set $Y_{0}$ with complement $Y_{1}$, such that $Y_{0} \cap D_{\alpha}(Y)$ contains at least $n-1$ points, and $Y_{1} \cap D_{\alpha}(Y) \neq \varnothing$. By the induction hypothesis there are continuous functions mapping $Y_{0}$ onto $\left[0, \omega^{\alpha}(n-1)\right]$, and $Y_{1}$ onto $\left[\omega^{\alpha}(n-1)+1, \omega^{\alpha} n+1\right]$. Apply (1) to prove the existence of a continuous function from $Y$ onto $\omega^{\alpha} n+1$.

THEOREM 4. If $A$ and $B$ are countable superatomic Boolean algebras, then the following are equivalent:

(i) $\Gamma(A) \leqq \Gamma(B)$;

(ii) there is a monomorphism from $A$ to $B$;

(iii) there is an epimorphism from $B$ to $A$;

(iv) $A$ is a retract of $B$.

Proof. Let $X=\omega^{\alpha} m+1$ be the Boolean space of $A$, and $Y=$ $\omega^{\beta} n+1$ that of $B$, both with the order topology, $\alpha$ and $\beta$ countable ordinals, $m$ and $n$ natural numbers. Part (i) implies (ii) by Theorem 3. Assuming (ii), there is a continuous function $\theta$ from $Y$ onto $X$; $\theta\left[D_{\beta}(Y)\right]$ is finite and contains $D_{\beta}(X)$ by Lemma 1 , so (ii) implies (i). Part (i) implies (iii) because $X$ can be identified with the initial segment of $Y$, and (iii) implies (i) by Lemma 2. Finally, (iii) is equivalent to (iv) because $A$ is countable and therefore projective [3].

If $A$ and $B$ are superatomic Boolean algebras and $A$ is countable, then an epimorphism from $B$ to $A$ would insure the existence of a monomorphism from $A$ to $B$. However, there are superatomic Boolean 
algebras $A$ and $B, A$ countable, and $\Gamma(A) \leqq \Gamma(B)$, for which there is no epimorphism from $B$ to $A$.

EXAMPLE 5. Let $N$ be the set of natural numbers with the discrete topology. Pick an uncountable family of subsets of $N$ such that each pair intersect in at most a finite number of points. (See [5].) Extend this family to a family $\mathscr{F}=\left\{F_{i}: i \in I\right\}$, indexed by some set $I$, maximal with respect to the property that every pair has a finite intersection. Let $J \subseteq I$ be the set of indices for which $F_{i}$ is infinite. Adjoin to $N$ a set $\left\{x_{i}: i \in J\right\}$ of new points, and take the family of all sets $U \cup\left\{x_{i}\right\}$, where $U$ is a co-finite subset of $F_{i}$, as open neighborhoods of $x_{i}, i \in J$. Let $X$ be the one-point compactification, by the point $x_{0}$, of the resulting topological space. It is easily seen that $X$ is Hausdorff, and has a basis of clopen sets. Moreover, $D_{1}(X)$ is the one-point compactification of the set of its isolated points $\left\{x_{i}: i \in J\right\}$, so the Boolean algebra associated with $X$ is superatomic with cardinal sequence $\boldsymbol{S}_{0},|J|, 1$.

Suppose that there is a homeomorphism $\theta$ of $\omega^{2}+1$ onto $Y, Y$ a closed subspace of $X$. Since $D_{2}(Y) \subseteq D_{2}(X), \theta\left(\left\{\omega^{2}\right\}\right)=x_{0}$, and since $D_{1}(Y) \leqq D_{1}(X),\{\theta(\omega \cdot n): n<\omega\}$ is a countable infinite subset of $\left\{x_{i}\right.$ : $i \in J\}$. There are disjoint, clopen subsets $U_{n}, n<\omega$, in $X$ such that $U_{n} \cap D_{1}(X)=\{\theta(\omega \cdot n)\}$, and $U_{n} \cap Y=\left[U_{n} \cap Y \cap N\right] \cup\{\theta(\omega \cdot n)\}$ is infinite for all $n<\omega$. Pick some point $y_{n} \in U_{n} \cap Y \cap N$ for each $n<\omega$. By the maximality assumption, there is a set $F_{i} \in \mathscr{F}$ for which $F_{i} \cap\left\{y_{n}: n<\omega\right\}$ is infinite. Then $x_{i} \notin Y$, but $x_{i}$ is an accumulation point of $Y$, so $Y$ was not closed in $X$.

Terminology. For the rest of the paper, $\mathscr{S}$ will designate the class of superatomic Boolean algebras. The infinite countable free Boolean algebra will be denoted $F_{0}$. In Theorems 7, 8, 9, and 10, $\mathscr{C}$ will be a nonempty class of infinite, countable Boolean algebras, closed under isomorphisms. The finite Boolean algebras will not be considered, because the situation in this case is well known. A finite Boolean algebra is a retract of every Boolean algebra of larger cardinality.

Lemma 6. If $C$ is a countable, infinite, nonsuperatomic Boolean algebra, then every countable Boolean algebra $A$ is a retract of $C$.

Proof. Because $C$ is nonsuperatomic, $C$ has an atomless homomorph. Every countable, infinite, atomless Boolean algebra is isomorphic to $F_{0}$. (See [2].) Since every countable Boolean algebra $A$ is a homomorph of $F_{0}, A$ is a retract of $C$. 
Theorems 7 through 10 follow readily from Theorems 3 and 4, Lemma 6, and the facts: (A) Every countable Boolean algebra is projective. (B) A Boolean algebra is superatomic if and only if it has no subalgebra isomorphic to $F_{0}$.

THEOREM 7. Let $\mathscr{A}$ be the class of Boolean algebras which have a subalgebra in $\mathscr{C}$.

(i) If $\mathscr{C}$ contatins no superatomic Boolean algebras then $\mathscr{A}$ is the class of all nonsuperatomic Boolean algebras.

(ii) If $C_{0}$ is the superatomic Boolean algebra in $\mathscr{C}$ with the smallest cardinal sequence, then $\mathscr{A}$ is the class of all Boolean algebras except $\left\{A \in \mathscr{S}: \Gamma(A) \nsupseteq \Gamma\left(C_{0}\right)\right\}$.

Theorem 8. Let $\mathscr{A}$ be the class of subalgebras of members of $\mathscr{C}$.

(i) If $\mathscr{C}$ contains a nonsuperatomic Boolean algebra $C_{0}$, then $\mathscr{A}$ is the class of all countable Boolzan algebras.

(ii) If $\mathscr{C}$ is a class of superatomic Boolean algebras, then $\mathscr{A}$ is the class of countable superatomic Boolean algebras $A$ for which $\Gamma(A) \leqq \Gamma(C)$ for some $C \in \mathscr{C}$.

Theorem 9. The class of all homomorphs of members of $\mathscr{C}$ is just the class of all subalgebras of members of $\mathscr{C}$.

THeorem 10. Let $\mathscr{A}$ be the class of Boolean algebras which have a homomorph in $\mathscr{C}$.

(i) If $\mathscr{C}$ contains no superatomic Boolean algebras, then $\mathscr{A}$ is the class of Boolean algebras with a countable infinite, free retract.

(ii) If $C_{0}$ is the superatomic Boolean algebra in $\mathscr{C}$ with the least cardinal sequence, then $\mathscr{A}$ is the class of Boolean algebras which have $C_{0}$ as a retract.

The general problem of determining when a Boolean algebra is a retract of another seems to be quite difficult. The situation is not simple even in the case of superatomic Boolean algebras, as was seen in Example 5. Some results are obtained when attention is restricted to the field of all finite and co-finite subsets of a countable infinite set, however.

Let $P_{0}$ denote the power set on a countable, infinite set, and let $T_{0}$ be the quotient of $P_{0}$ by the ideal generated by its atoms. Let $S_{0}$ be the field of all finite and co-finite subsets of a countable, infinite set. If $B$ is a subalgebra of a Boolean algebra $A$, a simple extension of $B$ in $A$ is a subalgebra of $A$ generated by the set $\{a\} \cup B$, where $a$ is some element in $A$. 
THEOREM 11. A Boolean algebra $A$ has no countable, infinite homomorph if and only if for every infinite homomorph $B$ of $A$, there is an epimorphism from $B$ to a subalgebra $C$ of $T_{0}$ such that every simple extension of $C$ in $T_{0}$ is atomless.

Proof. By Theorem 10, a Boolean algebra $A$ has no countable, infinite homomorph if and only if $S_{0}$ is not a homomorph of $A$.

Suppose that $S_{0}$ is not a homomorph of $A$, and that $B$ is an infinite homomorph. Pick a set $\left\{b_{n}: n<\omega\right\}$ of disjoint elements in $B$. Let $D$ be the quotient of $B$ by an ideal maximal with respect to not containing any element $b_{n}, n<\omega$. Then $D$ is an infinite atomic Boolean algebra with a countable set $X$ of atoms. The completion $P$ of $D$ is isomorphic to the power set on $X$, and we shall consider $D$ as a subalgebra of $P$. Let $C$ be the quotient $D /(X)$ of $D$ by the ideal generated by $X$, and notice that $C$ can be embedded in $P /(X)$. This latter quotient is isomorphic to $T_{0}$.

Suppose $a$ is an atom in a simple extension of $C$ in $T_{0}$. Then every member of $C$ either contains $\{a\}$ or is disjoint from $\{a\}$. This can happen in the quotient algebras only when there is an infinite set $Y$ of atoms in $D$, and every element of $D$ contains either a finite or co-finite subset of $Y$. Thus the quotient of $D$ by the ideal of all elements disjoint from the members of $Y$ is isomorphic to $S_{0}$, but also is a homomorph of $A$.

Conversely, if $A$ has an infinite, countable homomorph, then $S_{0}$ is also a homomorph. Every homomorph of $S_{0}$ is atomic, so there can be no epimorphism from $S_{0}$ to a subalgebra of $T_{0}$ with every simple extension atomless.

\section{REFERENCES}

1. G. W. Day, Superatomic Boolean algebras, Pacific J. Math. 23 (1967), 479-489.

2. Ph. Dwinger, Introduction to Boolean algebras, Wurzburg, 1961.

3. P. R. Halmos, Injective and projective Boolean algebras, Proc. Sympos. Pure Math. 2 (1961), 114-122.

4. R. D. Mayer and R. S. Pierce, Boolean algebras with ordered bases, Pacific J. Math. 10 (1960), 925-942.

5. W. Sierpinski, Cardinal and Ordinal Numbers, Warsaw, 1958.

Received November 5, 1969, and in revised form January 23, 1970. This work is an amplification of part of the author's doctoral dissertation submitted to the University of Washington.

UNIVERSITY OF BRITISH COLUMBIA

VANCOUVer, British Columbia 


\section{PACIFIC JOURNAL OF MATHEMATICS}

\section{EDITORS}

\author{
H. SAMELSON \\ Stanford University \\ Stanford, California 94305

\section{Richard Pierce} \\ University of Washington \\ Seattle, Washington 98105
}

J. DugundJI

Department of Mathematics

University of Southern California

Los Angeles, California 90007

RichaRd ARENS

University of California

Los Angeles, California 90024

\section{ASSOCIATE EDITORS}
E. F. BECKENBACH
B. H. NeUMANN
F. WOLE
K. YoshidA

\section{SUPPORTING INSTITUTIONS}

\author{
UNIVERSITY OF BRITISH COLUMBIA \\ CALIFORNIA INSTITUTE OF TECHNOLOGY \\ UNIVERSITY OF CALIFORNIA \\ MONTANA STATE UNIVERSITY \\ UNIVERSITY OF NEVADA \\ NEW MEXICO STATE UNIVERSITY \\ OREGON STATE UNIVERSITY \\ UNIVERSITY OF OREGON \\ OSAKA UNIVERSITY \\ UNIVERSITY OF SOUTHERN CALIFORNIA
}

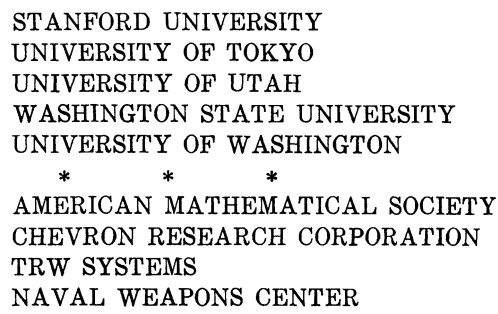

The Supporting Institutions listed above contribute to the cost of publication of this Journal, but they are not owners or publishers and have no responsibility for its content or policies.

Mathematical papers intended for publication in the Pacific Journal of Mathematics should be in typed form or offset-reproduced, (not dittoed), double spaced with large margins. Underline Greek letters in red, German in green, and script in blue. The first paragraph or two must be capable of being used separately as a synopsis of the entire paper. The editorial "we" must not be used in the synopsis, and items of the bibliography should not be cited there unless absolutely necessary, in which case they must be identified by author and Journal, rather than by item number. Manuscripts, in duplicate if possible, may be sent to any one of the four editors. Please classify according to the scheme of Math. Rev. Index to Vol. 39. All other communications to the editors should be addressed to the managing editor, Richard Arens, University of California, Los Angeles, California, 90024.

50 reprints are provided free for each article; additional copies may be obtained at cost in multiples of 50 .

The Pacific Journal of Mathematics is published monthly. Effective with Volume 16 the price per volume (3 numbers) is $\$ 8.00$; single issues, $\$ 3.00$. Special price for current issues to individual faculty members of supporting institutions and to individual members of the American Mathematical Society: $\$ 4.00$ per volume; single issues $\$ 1.50$. Back numbers are available.

Subscriptions, orders for back numbers, and changes of address should be sent to Pacific Journal of Mathematics, 103 Highland Boulevard, Berkeley, California, 94708.

PUBLISHED BY PACIFIC JOURNAL OF MATHEMATICS, A NON-PROFIT CORPORATION

Printed at Kokusai Bunken Insatsusha (International Academic Printing Co., Ltd.), 7-17, Fuj̣imi 2-chome, Chiyoda-ku, Tokyo, Japan. 


\section{Pacific Journal of Mathematics}

\section{Vol. 35, No. $2 \quad$ October, 1970}

Valentin Danilovich Belousov and Palaniappan L. Kannappan, Generalized Bol functional equation .................................... 259

Charles Morgan Biles, Gelfand and Wallman-type compactifications ........... 267

Louis Harvey Blake, A generalization of martingales and two consequent convergence theorems .................................... 279

Dennis K. Burke, On p-spaces and $w \Delta$-spaces..................... 285

John Ben Butler, Jr., Almost smooth perturbations of self-adjoint operators . . . . . . 297

Michael James Cambern, Isomorphisms of $C_{0}(Y)$ onto $C(X) \ldots \ldots \ldots \ldots \ldots . \ldots 307$

David Edwin Cook, A conditionally compact point set with noncompact closure ... 313

Timothy Edwin Cramer, Countable Boolean algebras as subalgebras and homomorphs .........................................

John R. Edwards and Stanley G. Wayment, A v-integral representation for linear operators on spaces of continuous functions with values in topological vector spaces.............................................

Mary Rodriguez Embry, Similarities involving normal operators on Hilbert

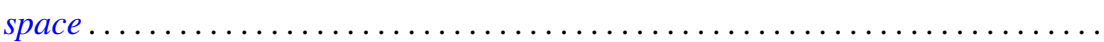

Lynn Harry Erbe, Oscillation theorems for second order linear differential

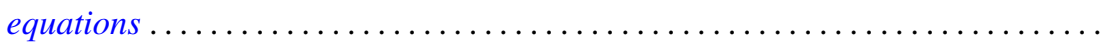

William James Firey, Local behaviour of area functions of convex bodies .......... Joe Wayne Fisher, The primary decomposition theory for modules ..............

Gerald Seymour Garfinkel, Generic splitting algebras for Pic ..................

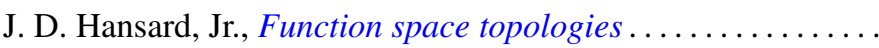

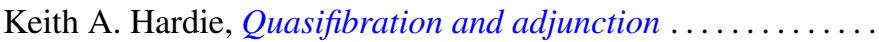

G. Hochschild, Coverings of pro-affine algebraic groups ...........

Gerald L. Itzkowitz, On nets of contractive maps in uniform spaces ..

381

389

399

417

Melven Robert Krom and Myren Laurance Krom, Groups with free nonabelian subgroups....................................

James Robert Kuttler, Upper and lower bounds for eigenvalues by finite differences ......................................

Dany Leviatan, A new approach to representation theory for convolution transforms . . .

Richard Beech Mansfield, Perfect subsets of definable sets of real numbers ...

Brenda MacGibbon, A necessary and sufficient condition for the embedding of a

Lindelof space in a Hausdorff $\mathscr{H} \sigma$ space ..................

David G. Mead and B. D. McLemore, Ritt's question on the Wronskian ....

Edward Yoshio Mikami, Focal points in a control problem .....

Paul G. Miller, Characterizing the distributions of three independent n-dimensional random variables, $X_{1}, X_{2}, X_{3}$, having analytic characteristic functions by the joint distribution of $\left(X_{1}+X_{3}, X_{2}+X_{3}\right)$. . .

P. Rosenthal, On the Bergman integral operator for an elliptic partial differential equation with a singular coefficient....

Douglas B. Smith, On the number of finitely generated $O$-group 